\title{
Holographic vortices in the presence of dark matter sector
}

\section{Marek Rogatko and Karol I. Wysokinski}

Institute of Physics, Maria Curie-Sktodowska University, 20-031 Lublin, pl. Marii Curie-Sktodowskiej 1, Poland

E-mail: rogat@kft.umcs.lublin.pl, karol.wysokinski@umcs.pl

ABSTRACT: The dark matter seem to be an inevitable ingredient of the total matter configuration in the Universe and the knowledge how the dark matter affects the properties of superconductors is of vital importance for the experiments aimed at its direct detection. The homogeneous magnetic field acting perpendicularly to the surface of $(2+1)$ dimensional s-wave holographic superconductor in the theory with dark matter sector has been modeled by the additional U(1)-gauge field representing dark matter and coupled to the Maxwell one. As expected the free energy for the vortex configuration turns out to be negative. Importantly its value is lower in the presence of dark matter sector. This feature can explain why in the Early Universe first the web of dark matter appeared and next on these gratings the ordinary matter forming cluster of galaxies has formed.

KEYwords: Holography and condensed matter physics (AdS/CMT), AdS-CFT Correspondence, Black Holes

ArXIV EPRINT: 1510.06137 


\section{Contents}

1 Introduction 1

2 Superconductor with dark matter sector in AdS/CFT theory 4

$\begin{array}{lll}3 & \text { Leading orders of the equations of motion } & 7\end{array}$

$\begin{array}{lll}3.1 \text { Zeroth order } & 7\end{array}$

3.2 Next to zeroth order 8

$\begin{array}{lll}3.2 .1 & \text { Scalar field equation } & 8\end{array}$

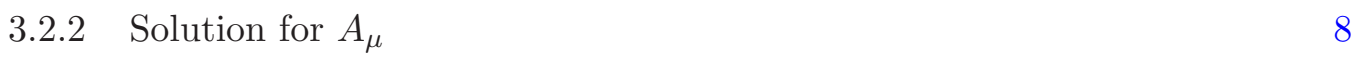

$\begin{array}{lll}3.2 .3 \text { Current } & 9\end{array}$

$\begin{array}{lll}4 & \text { Free energy } & 10\end{array}$

5 Superconducting coherence length $\quad 13$

6 London equation with dark matter sector 16

$\begin{array}{lll}7 & \text { Conclusions } & 19\end{array}$

\section{Introduction}

Proposed as the equivalence between type IIB superstring theory on $A d S_{5} \times S^{5}$ spacetime and $\mathcal{N}=4 \mathrm{SU}(N)$ supersymmetric Yang Mills theory on $(3+1)$-dimensional boundary [1][3] and generalized to the other gravitational backgrounds [4], the gauge/gravity duality helps us to understand the physics of strongly correlated systems.

The main feature of the string theories is that gravity emerges from them in a natural way. In this context the string theories dominated by the classic gravity configurations provide a basis for the weak-strong duality [5]. In technical terms [6] it means that the partition function of the field theory $Z[A(\mathrm{x}, t)]$, where $A(\mathrm{x}, t)$ is the source field that couples to the currents $\mathbf{j}(\mathbf{x}, t)$, equals to that of the gravity dual $Z[A(\mathbf{x}, r, t)]$ with the boundary condition $\lim _{r \rightarrow \infty} A(\mathbf{x}, r, t)=A(\mathbf{x}, t)$. The latter partition function is prevailed by the classical configurations and thus given by the exponential of the classical action of the aforementioned configurations.

The AdS/CFT correspondence has been successfully applied for the description of the superconducting phase transition of the single s-wave superconductor [7], as well as, the other symmetries [8]-[26]. The effects of the higher order curvatures [27]-[32] of the gravity background and non-linearities in the electrodynamic theory [33]-[36] have been widely studied. 
The aforementioned studies were also extended by the modification of gravity theory by considering the five-dimensional AdS solitonic metric [37], which enables to construct a model of the holographic insulator/superconductor phase transition at zero temperature [38]. The AdS soliton line element dual to a confined field theory with a mass gap, imitates an insulator phase [39].

The effects of magnetic field (which will be crucial in our further studies) on holographic superconductors were also intensively studied. It was revealed that by adding magnetic charge to a black hole, the holographic superconductor could be immersed in magnetic field [40]. The studies of the problem in question were also conducted in Gauss Bonnet gravity and in Weyl-corrected and non-linear electrodynamics [41]-[44]. Holographic vortices and droplets influenced by magnetic field were elaborated in [45]-[46], while the vector condensation generated by this factor was studied in [19, 47]. On the other hand, the magnetic penetration depth was computed in [7], where it was envisaged that the holographic superconductors are of type II. In order to find the superconducting coherence length and magnetic penetration depth [48] one perturbs the AdS-Schwarzschild system mimicking s-wave superconductor near the critical temperature. These works triggered the investigations in p-wave as well as d-wave holographic superconductors [49]-[50]. It has been found that the superconducting coherence length was proportional to $\left(1-T / T_{c}\right)^{-1 / 2}$ near the critical temperature. Similar dependence of the magnetic penetration depth $\lambda \propto\left(1-T / T_{c}\right)^{-1 / 2}$ has been observed. These results agree with the standard Ginzburg-Landau theory.

In [51] the interesting case of the Abelian Chern-Simons Higgs model in $(2+1)$ dimensions was elaborated. From this point of view the higher derivative corrections to the Abelian gauge sector in the bulk were studied [52]. The vortex lattice solution in a holographic superconductors constructed from a charged scalar condensate was elaborated in [53]. The perturbative solution near the second-order phase transition represents the holographic Abrikosov lattice. On the other hand, the holographic vortex-flow solution was given in [54].

The other crucial problem is the question about the possible matter configuration in the AdS spacetime. This problem was considered in the case of strictly stationary Einstein-Maxwell AdS spacetime [55] and its generalization to the low-energy limit of the heterotic string theory with arbitrary number of U(1)-gauge fields [56]. It was concluded that the spacetimes in question can not allow for the existence of nontrivial configurations of complex scalar or form fields.

The origin of cosmic structures can be understood in terms of evolution of matter perturbations arising after inflationary period. The dark matter constitutes the key ingredient in the aforementioned processes, as a potential wells where the ordinary matter accrete. If dark matter appears in the form of particles coupled to ordinary matter then a certain level of emitted radiation is expected. The non-gravitational signals are anticipated from interaction of dark matter with ambient medium, annihilation or decay by a direct emission or through particle production. The non-gravitational signals of it should be in principle proportional to the density of clumps of dark matter. Recently, a new method to constrain the process of elastic scattering between dark matter and the Standard Model particles in the Early Universe was proposed [57, 58]. 
Dark matter may also leave its footprints during collapse of neutron stars and in the first generation of stars [59]-[61]. On the other hand, the behavior of dark matter and dark energy collapse is studied numerically from the point of view of possible features of black hole and wormhole formations [62]-[63]. This is an important problem in studies of the black hole growth in the Early Universe.

The dark matter non-gravitational interactions, having implications for the particle physics beyond the Standard Model, triggered search for high-energy photons produced by annihilation which in turn causes the resurgence in inspection of gamma-rays emission from the dwarf galaxies [64]. This problem is also interesting in the context of planing varieties of gamma-rays telescopes in the range of energy from 1 to $100 \mathrm{MeV}$ [65]. Investigations of dilatonic like coupling to photons which can be caused by ultralight dark matter was reported in [66]. This process can induce oscillations in the fine-structure constant.

The dark matter sector model may have a strong support by some astrophysical data such as observation of $511 \mathrm{keV}$ gamma rays [67] experiments detecting the electron positron excess in galaxy [68, 69], and possible explanation of muon anomalous magnetic moment [70]. Recent astronomical observations reveal that collisions among galaxy clusters provide new tests of non-gravitational forces acting on dark matter [71, 72]. The experiments in question may disfavor some extensions of the Standard Model.

The idea that dark matter sector is charged under a new U(1)-gauge group and is coupled to the ordinary Maxwell field gained experimental verifications. The low-energy $e^{+} e^{-}$colliders offer the environment to probe low-energy mass dark sector [73]. The data from BABAR detector were revealed bounded with a search for dark photon production in the range of mass $0.02<m<10.2 \mathrm{GeV}$, but no significant signal has been observed. The other experiments are planned to cover the energy region $15 \leq m \leq 30 \mathrm{MeV}$.

It is thus obvious that study of dark matter and its observational consequences is both timely and of crucial importance. In this paper we add a novel aspect to it by analyzing the behavior of holographic superconductors.

We shall try to answer the question how a dark matter sector modifies the ordinary phase transitions known from the previous studies and what the role of the dark matter coupling constant $\alpha$, binding dark matter fields with ordinary Maxwell gauge field is. We investigate analytically the perturbation of the dual gravity theory to reveal the superconducting coherence length and magnetic penetration depth close to the superconducting phase transition point. The main aim of this paper is to study the influence of the dark matter sector on the holographic vortex. In the previous works [74]-[78], various aspects of phase transitions in s-wave and p-wave holographic superconductor theory with ordinary matter sector coupled to another U(1)-gauge field which describes dark matter have been elaborated. One may expect that dark matter authorizes a part of a larger particle sector interacting with the visible matter and not completely decoupled [79]-[85].

The other tantalizing question which should be answered is how to probe the early cosmological structures in the Universe with the AdS/CFT correspondence. The behaviour of the holographic superconductors in the external magnetic field reveals the fact that the condensation takes place easier in the presence of dark matter. Such mechanism could have been at play in the Early Universe and it may be applied for the explanation of the 
formation of the primordial components of the Universe which results in presently observed voids on which ordinary matter condensates.

We are mainly interested in the question how to detect the dark matter existing in our neighbourhood using the present day technology and the known superconductors. Namely, if the dark matter influences the superconductor as predicted, its presence may be detected by a cleverly designed and precise experiments with superconductors. This idea is as follows. If the structures with and without dark matter do exist in the Earth neighbourhood, they may be in principle detected in long time (a few years or so) experiments observing the yearly changes of the superconducting properties of materials, under the assumption that the time scale for the changes in dark matter distribution around the Sun is large enough [86].

In the present paper, the case of homogeneous magnetic field acting perpendicularly to the surface of s-wave holographic superconductor in the theory with dark matter sector has been considered. Dark matter has been modeled by the additional U(1)-gauge field coupled to Maxwell one and (2+1)-dimensional vortices have been studied in the considered theory. Free energy for the vortex configuration turns out to be negative and it is less for the configuration with dark matter sector than for ordinary matter case. One concludes that the dark matter sector vortex configurations are more stable than trivial configuration where there is no charge scalar condensate. On the other hand, because of the fact that dark matter vortices build less free energy configuration than ordinary matter ones, this fact can explain why in the Early Universe first form the web of dark matter and next on these tendrils the ordinary matter condenses forming cluster of galaxies.

The paper is organized as follows. In section 2 we describe the main features of the theory with dark matter section which is mimicked by the coupling between ordinary Maxwell and U(1)-gauge fields. In section 3, to the leading order, we find perturbatively solutions of the equations of motion in the underlying theory. Section 4 is devoted to free energy for the considered system which determines thermodynamics stability of the dark matter sector vortex. Section 5 is connected with calculations of the superconducting coherence length and magnetic penetration depth which depend on the $\alpha$-coupling constant of the dark matter part of the theory. In section 6 we find the analogy of the London equation for the holographic vortex in the theory in question and calculate the superfluid density and magnetic penetration depth. Both quantities are affected by the influence of the dark matter section of the considered theory.

\section{Superconductor with dark matter sector in AdS/CFT theory}

The gravitational action in $(3+1)$ dimensions is taken in the form

$$
S_{g}=\int \sqrt{-g} d^{4} x(R-2 \Lambda)
$$

where $\Lambda=-3 / L^{2}$ stands for the cosmological constant, while $L$ is the radius of the AdS spacetime. We shall examine the Abelian-Higgs sector coupled to the second U(1)-gauge 
field which mimics the dark matter sector of the underlying theory [83]. The adequate action incorporating dark matter is provided by

$$
\begin{aligned}
S_{m}=\int \sqrt{-g} d^{4} x( & -\frac{1}{4} F_{\mu \nu} F^{\mu \nu}-\left[\nabla_{\mu} \psi-i q A_{\mu} \psi\right]^{\dagger}\left[\nabla^{\mu} \psi-i q A^{\mu} \psi\right]-m^{2}|\psi|^{2} \\
& \left.-\frac{1}{4} B_{\mu \nu} B^{\mu \nu}-\frac{\alpha}{4} F_{\mu \nu} B^{\mu \nu}\right),
\end{aligned}
$$

where $F_{\mu \nu}=2 \nabla_{[\mu} A_{\nu]}$ stands for the ordinary Maxwell field strength tensor, while the second U(1)-gauge field $B_{\mu \nu}$ is given by $B_{\mu \nu}=2 \nabla_{[\mu} B_{\nu]}$. Moreover, $m, q$ represent, respectively a mass and a charge related to the scalar field $\psi . \alpha$ is a coupling constant between $\mathrm{U}(1)$ fields.

One should recall that the model in question is widely studied in the context of particle physics, in order to observe the signature of dark mater in planned polarized electron scattering experiments in the Large Hadron Collider [84, 85].

Varying the action with respect to the considered fields one obtains the equations of motion which can be written in the form as

$$
\begin{aligned}
\left(\nabla_{\mu}-i q A_{\mu}\right)\left(\nabla^{\mu}-i q A^{\mu}\right) \psi-m^{2} \psi & =0, \\
\tilde{\alpha} \nabla_{\mu} F^{\mu \nu} & =j^{\nu},
\end{aligned}
$$

where the current $j^{\nu}$ yields

$$
j^{\nu}=i\left[\psi^{\dagger}\left(\nabla^{\nu}-i q A^{\nu}\right) \psi-\psi\left(\nabla^{\nu}+i q A^{\nu}\right) \psi^{\dagger}\right]=0
$$

and $\tilde{\alpha}=1-\alpha^{2} / 4$. In the above relation we have used the equation determining $B_{\mu \nu}$ field

$$
\nabla_{\mu} B^{\mu \nu}+\frac{\alpha}{2} \nabla_{\mu} F^{\mu \nu}=0
$$

in order to eliminate this field and obtain the relation (2.4).

The presence of the dark matter sector is marked by the appearance of $\tilde{\alpha}$ in the equations of motion. Equation (2.6) is a good starting point to interpret the physical meaning of the parameter $\alpha$ and the superconducting phase transition as seen by the appearance of the Meissner effect (the mass of the photon). The spontaneous symmetry breaking makes the usual photon massive. The dark photons, more precisely the dark sector in our approach, modify only the properties of Maxwell electrodynamic in a way visible in the relation (2.6) and the parameter $\alpha$ describes these modifications. From this point of view the holographic $s$ and $p$-wave superconductor phase transitions were previously studied [74-76] in the presence of dark matter. It was revealed that there were some imprints of dark matter sector on holographic phase transitions. In particular it has been found [76] that in the probe limit the prefactor $\Gamma$ between the value of the condensing operator in the relation $\langle\mathcal{O}\rangle=\Gamma \sqrt{1-T / T_{c}}$ does depend on $\alpha . \quad \Gamma \propto \sqrt{\tilde{\alpha}}$. Neither the superconducting transition temperature nor critical value of the chemical potential for insulator to metal transition depend on $\alpha$. Much more severe dependence on the dark 
matter coupling has been observed in the backreacting theory [74], where the transition temperature depends on $\alpha$. The p-wave superconductors described by the SU(2) Yang-Mills theory features inter alia the increase [77] of the transition temperature with increase of the coupling $\alpha$. On the other hand, the critical chemical potential $\mu_{c}$ for the quantum phase transition between insulator and a metal is a decreasing function of $\alpha$.

In the theory with the action given by equations (2.1) and (2.2) the gravitational background will be provided by the four-dimensional AdS-Schwarzschild black hole background of the form

$$
d s^{2}=-f(r) d t^{2}+\frac{1}{f(r)} d r^{2}+\frac{r^{2}}{L^{2}}\left(d x^{2}+d y^{2}\right),
$$

where the metric function reads

$$
f(r)=\frac{r^{2}}{L^{2}}\left(1-\frac{r_{+}^{3}}{r^{3}}\right) .
$$

$\mathrm{L}$ is the radius of the AdS spacetime and $r_{+}$is the horizon of the black hole. The Hawking temperature of the black hole is found to be

$$
T=\frac{3 r_{+}}{4 \pi L^{2}} .
$$

In what follows, without loss of generality, we shall set the spacetime radius $L$ and the charge related to the scalar field equal to 1 . One also changes the variables replacing the space coordinate $r$ by $u=r_{+} / r$. This replacement results in the line element of the form

$$
d s^{2}=-f(u) d t^{2}+\frac{r_{+}^{2}}{u^{4} f(u)} d u^{2}+\frac{r_{+}^{2}}{u^{2}}\left(d x^{2}+d y^{2}\right)
$$

where $f(u)=r_{+}^{2} / u^{2}\left(1-u^{3}\right)$.

We are interested in the properties of superconductors subject to the external magnetic field. As it is well known from experiments on superconducting systems and in agreement with Ginzburg-Landau theory, type I superconductors are characterized by the single value of the critical magnetic field $B_{c}$, beyond which the superconductivity is destroyed. Such systems are characterized by the penetration depth $\lambda$ smaller than the superconducting coherence length $\xi$. The existence of the border between normal metal and superconductor increases its energy and makes the system thermodynamically unstable. On the other hand, in the opposite limit of $\lambda / \xi \gg 1$ (type II superconductors) the existence of the border decreases the energy and allows the magnetic field to penetrate into the metal in the form of vortices. The process starts at the "lower magnetic field" $B_{c 1}$ and continues until "upper magnetic field" $B_{c 2}$, when the vortices are so dense that they overlap and the system undergoes superconductor to normal metal transition. The vortices are normal systems at their cores. We do not consider here the dynamics of the gauge field. It is important in analysis of various properties of superconductors [87, 88] in particular the Meissner effect. Detailed studies of superfluid and superconductor vortices were given in [89], where the conditions under a type I or type II superconductor can be achieved. The computations were at the fully nonlinear level and included the gravitational backreaction. 
One should remark that in the considered theory there are in principle two ways of choosing the magnetic field. One is the ordinary magnetic field introduced by the appropriate component of the U(1)-gauge Maxwell field, $A_{y}$. The other, is to choose the magnetic field of dark matter sector and then by the equation of motion (2.6) receive the Maxwell component of $A_{y}$ which enters the relation (2.4). Choosing the second possibility we assume constant dark matter magnetic field $\hat{B}$

$$
B_{y}=\hat{B} x .
$$

Thus, having in mind equation (2.6), we obtain

$$
A_{y}=\frac{2}{\alpha}\left(c_{1}-\hat{B}\right) x+c_{2}
$$

where $c_{1,2}$ are arbitrary constants. In our further considerations we shall keep the general form of $A_{y}$ and in the resulting conclusion one specifies the influence of the two aforementioned possibilities of choosing magnetic field.

To proceed further we shall solve the equations of motion perturbatively in the probe limit. Namely, we define the deviation parameter $\epsilon_{H}=\left(B_{c 2}-B\right) / B_{c 2}$, with the property that $\left|\epsilon_{H}\right| \ll 1$, and expand gauge fields $A_{\mu}(\mathbf{x}, u)$, the scalar field $\psi(\mathbf{x}, u)$ and the current $j_{\mu}(\mathbf{x}, u)$ in series of $\epsilon_{H}$

$$
\begin{aligned}
A_{\mu}(\mathbf{x}, u) & =A_{\mu}^{(0)}(\mathbf{x}, u)+\epsilon_{H} A_{\mu}^{(1)}(\mathbf{x}, u)+\ldots, \\
\psi(\mathbf{x}, u) & =\epsilon_{H}^{\frac{1}{2}} \psi_{1}(\mathbf{x}, u)+\epsilon_{H}^{\frac{3}{2}} \psi_{2}(\mathbf{x}, u)+\ldots \\
j_{\mu}(\mathbf{x}, u) & =\epsilon_{H} j_{\mu}^{(1)}(\mathbf{x}, u)+\epsilon_{H}^{2} j_{\mu}^{(2)}(\mathbf{x}, u)+\ldots,
\end{aligned}
$$

where $\mathbf{x}=(\mathbf{x}, \mathbf{y})$. The above chosen expansions are analogous to that used previously [4854]. The different powers of $\epsilon_{H}$ are related to the equations of motion for the scalar field $\psi$ and $A_{t}$ as discussed in [48]. They envisage the fact that the scalar field $\psi$ acts as an order parameter and vanishes at the critical value of the magnetic field in the mean field like manner. In what follows we solve and analyze the solutions of the equations (2.3) and (2.4), for $A_{\mu}$ and $\psi$, up to the leading order.

\section{Leading orders of the equations of motion}

\subsection{Zeroth order}

Substituting the relations (2.13)-(2.15) into (2.3) and (2.4), we obtain the zeroth order equations of motion

$$
\tilde{\alpha} \nabla_{\nu} F^{\nu \mu(0)}=0 .
$$

In accordance with the assumed dependence of the fields in question on the parameter $\epsilon_{H}$, in zeroth order only the components of the gauge fields have non-zero values. In order to find the solutions we consider the ansatz as follows:

$$
A_{\mu}^{(0)}(\mathbf{x}, u)=\left(A_{t}(\mathbf{x}, u), A_{u}(\mathbf{x}, u), A_{x}(\mathbf{x}, u), A_{y}(\mathbf{x}, u)\right)=\left(\varphi(u), 0,0, A_{y}^{(0)}\right) .
$$


The zeroth order solution generates the chemical potential $\mu$ and the critical magnetic field $B_{c 2}$ as given by the relations

$$
A_{t}^{(0)}=\mu(1-u), \quad A_{u}^{(0)}, \quad A_{x}^{(0)}=0, \quad A_{y}^{(0)}=B_{c 2} x .
$$

The solution agrees with the fact that at $B=B_{c 2}$ the superconducting order parameter and the currents vanish.

\subsection{Next to zeroth order}

In this subsection one derives equations of motion for the scalar field $\psi$, the Maxwell gauge potentials $A_{\mu}$ and the current. As it follows from the assumed expansion, the non-zero value of the condensate $\psi_{1}$ is a coefficient in front of the $\sqrt{\epsilon_{H}}$ while the current being proportional to "square" of the scalar field appears in the order $\epsilon_{H}$. This provides a posteriori justification of the expansion (2.13)-(2.15).

\subsubsection{Scalar field equation}

Up to the $\sqrt{\epsilon_{H}}$-order the equation of motion for the scalar field can be written as

$$
\partial_{u}\left[f(u) \partial_{u} \psi_{1}\right]+\frac{1}{u^{2}}\left(\Delta-2 i A_{y}^{(0)} \partial_{y}-\left(A_{y}^{(0)}\right)^{2}\right) \psi_{1}+\frac{r_{+}^{2} \varphi^{2}(u)}{f(u) u^{4}} \psi_{1}-\frac{m^{2} r_{+}^{2}}{u^{4}} \psi_{1}=0
$$

In order to solve the above equations one separates the variables

$$
\psi_{1}(u, \mathbf{x})=\rho_{0}(u) H(\mathbf{x})=\rho_{0}(u) e^{i k_{y} y} X(x) .
$$

Inserting the above ansatz for $\psi_{1}(u, \mathbf{x})$ we arrive at the set of equations provided by

$$
\begin{aligned}
\rho_{0}^{\prime \prime}(u)+\frac{f^{\prime}(u)}{f(u)} \rho_{0}^{\prime}(u)+\frac{r_{+}^{2} \varphi^{2}(u)}{f(u) u^{4}} \rho_{0}(u)-\frac{m^{2} r_{+}^{2}}{u^{4}} \rho_{0}(u) & =\frac{\rho_{0}(u)}{\zeta^{2} u^{2} f(u)}, \\
-X^{\prime \prime}(x)+\left(A_{y}^{(0)}\right)^{2}\left(x-\frac{k_{y}}{A_{y}^{(0)}}\right)^{2} X(x) & =\frac{X(x)}{\zeta^{2}}
\end{aligned}
$$

where by $\zeta^{2}$ we denoted a separation constant.

\subsubsection{Solution for $A_{\mu}$}

With the choice of gauge in the form $A_{u}=0$, and assuming that the scalar field is the real one, the generalized Maxwell type equations at first order in $\epsilon_{H}$ are provided by

$$
\begin{aligned}
D^{(t)} A_{t}^{(1)} & =2 \frac{A_{t}^{(0)}\left|\psi_{1}\right|^{2} r_{+}^{2}}{\tilde{\alpha} u^{2}}, \\
D^{(i)} A_{m}^{(1)}-\partial_{m}\left(\delta^{f k} \partial_{f} A_{k}^{(1)}\right) & =\frac{j_{m}^{(1)} r_{+}^{2}}{\tilde{\alpha} u^{2}} \\
\partial_{u}\left(\delta^{a c} \partial_{a} A_{c}^{(1)}\right) & =0
\end{aligned}
$$


where the differential operators are denoted by

$$
D^{(t)}=u^{2} f(u) \partial_{u}^{2}+\Delta, \quad D^{(i)}=\partial_{u}\left(u^{2} f(u) \partial_{u}\right)+\Delta .
$$

By $\Delta$ we denoted the two-dimensional Laplacian, $\Delta=\partial_{x}^{2}+\partial_{y}^{2}$. As mentioned in [53], beside the gauge transformation equaling $u$-component of $\mathrm{U}(1)$-gauge field to zero also there exist transformation of the form $A_{m} \rightarrow A_{m}-\partial_{m} K(\mathbf{x})$ (the so-called residual transformation). It can be seen that $\left\langle J_{\mu}>\propto F_{u \mu}\right.$ is invariant under the transformation in question. This transformation allows us to set $\delta^{a c} \partial_{a} A_{c}^{(1)}=0$ and get the following equations instead of (3.8)-(3.10) [53]. Namely, one obtains

$$
\begin{aligned}
& D^{(t)} A_{t}^{(1)}=2 \frac{r_{+}^{2} A_{t}^{(0)}}{\tilde{\alpha} u^{2}} \rho_{0}^{2}(u) \sigma(\mathbf{x}), \\
& D^{(i)} A_{x}^{(1)}=\frac{r_{+}^{2}}{\tilde{\alpha} u^{2}} \rho_{0}^{2}(u) \epsilon_{x}^{y} \partial_{y} \sigma(\mathbf{x}), \\
& D^{(i)} A_{y}^{(1)}=\frac{r_{+}^{2}}{\tilde{\alpha} u^{2}} \rho_{0}^{2}(u) \epsilon_{y}^{x} \partial_{x} \sigma(\mathbf{x}),
\end{aligned}
$$

where $\epsilon_{a b}=\epsilon_{b}^{a}$ is the two-dimensional totally antisymmetric symbol with the properties $\epsilon_{x y}=-\epsilon_{y x}=1$. In the above relations we have introduced $\sigma(\mathbf{x})=|H(\mathbf{x})|^{2}$ represents the density of the condensate per unit volume around the point $\mathbf{x}$.

The boundary conditions for the equation (3.8) are given by demanding that $A_{t}^{(1)}$ is equal zero at $u=0$ and for $u=1$. For the equation (3.9) we impose that the solution is regular on the event horizon and $\epsilon_{H} F_{x y}^{(1)}$ calculated at the boundary of the AdS spacetime as given by the difference between magnetic and critical magnetic field $B-B_{c 2}$.

The formal solutions of the above relations can be cast in the form as

$$
\begin{aligned}
& A_{t}^{(1)}=-2 \frac{r_{+}^{2}}{\tilde{\alpha}} \int_{0}^{1} d u^{\prime} \frac{\rho_{0}^{2}\left(u^{\prime}\right)}{u^{\prime 2}} A_{t}^{(0)} \int d \mathbf{x}^{\prime} G_{B}\left(u, u^{\prime} \mid \mathbf{x}-\mathbf{x}^{\prime}\right) \sigma\left(\mathbf{x}^{\prime}\right), \\
& A_{m}^{(1)}=a_{m}(\mathbf{x})-\frac{r_{+}^{2}}{\tilde{\alpha}} \epsilon_{m}^{j} \int_{0}^{1} d u^{\prime} \frac{\rho_{0}^{2}\left(u^{\prime}\right)}{u^{\prime 2}} \int d \mathbf{x}^{\prime} G_{s}\left(u, u^{\prime} \mid \mathbf{x}-\mathbf{x}^{\prime}\right) \partial_{j} \sigma\left(\mathbf{x}^{\prime}\right),
\end{aligned}
$$

where the Green functions connected with the time and spatial components of the Maxwell gauge potentials imply

$$
\begin{aligned}
D^{(t)} G_{B}\left(u, u^{\prime} \mid \mathbf{x}-\mathbf{x}^{\prime}\right) & =-\delta\left(u-u^{\prime}\right) \delta\left(\mathbf{x}-\mathbf{x}^{\prime}\right), \\
D^{(i)} G_{s}\left(u, u^{\prime} \mid \mathbf{x}-\mathbf{x}^{\prime}\right) & =-\delta\left(u-u^{\prime}\right) \delta\left(\mathbf{x}-\mathbf{x}^{\prime}\right),
\end{aligned}
$$

with the AdS boundary conditions provided by the following:

$$
\begin{aligned}
\left.G_{B}\left(u, u^{\prime} \mid \mathbf{x}-\mathbf{x}^{\prime}\right)\right|_{u=0} & =\left.G_{B}\left(u, u^{\prime} \mid \mathbf{x}-\mathbf{x}^{\prime}\right)\right|_{u=1}=0 \\
\left.G_{s}\left(u, u^{\prime} \mid \mathbf{x}-\mathbf{x}^{\prime}\right)\right|_{u=0} & =\left.G_{s}\left(u, u^{\prime} \mid \mathbf{x}-\mathbf{x}^{\prime}\right)\right|_{u=1}=0 .
\end{aligned}
$$

\subsubsection{Current}

The AdS prescription enables us to find the U(1)-gauge fields current

$$
<J^{\beta}>=\left.\frac{\delta S_{\text {on-shell }}}{\delta A_{\beta}}\right|_{u \rightarrow 0}=\left.\left(F^{\beta u}+\frac{\alpha}{2} B^{\beta u}\right)\right|_{u \rightarrow 0} .
$$


Using equation (3.16) for $A_{j}^{(1)}$ and $F_{j u}^{(1)}=-\partial_{u} A_{j}^{(1)}$ we arrive at the folowing relation for the spatial components of the current in question

$$
<J_{i}>^{\mathrm{DM}}=\left.\left(F_{i u}+\frac{\alpha}{2} B_{i u}\right)\right|_{u \rightarrow 0} .
$$

In order to evaluate it let us consider the $u$-component of the first order expansions of the gauge fields in equation of motion (2.6). It implies

$$
\partial_{i}\left[\sqrt{-g}\left(B^{i u(1)}+\frac{\alpha}{2} F^{i u(1)}\right)\right]=0 .
$$

Having in mind the line element describing our spacetime we obtain the relation for the covariant components of the gauge fields provided by

$$
B_{i u}^{(1)}+\frac{\alpha}{2} F_{i u}^{(1)}=\frac{c_{i} r_{+}}{u^{2} f(u)},
$$

where $c_{i}$ are constants bounded with $x$ or $y$ components, respectively. Next, extracting $B_{i u}^{(1)}$ from the last equation and inserting it into $<J_{i}>^{\mathrm{DM}}$ we get

$$
<J_{i}>^{\mathrm{DM}}=\left.\tilde{\alpha} F_{i u}^{(1)}\right|_{u \rightarrow 0}+\beta_{i}(\alpha),
$$

where we set

$$
\beta_{i}(\alpha)=\frac{\alpha c_{i}}{2 r_{+}}
$$

By virtue of the above one finally arrives at the expression for the U(1)-gauge current in the theory with dark matter sector. Calculating $F_{i u}^{(1)}$ using equation (3.16) implies

$$
<J_{i}>^{\mathrm{DM}}=\left.\epsilon_{i}^{m} \partial_{m} \Theta(\mathbf{x})\right|_{u \rightarrow 0}+\beta_{i}(\alpha)
$$

where we have denoted

$$
\Theta(\mathbf{x})=\left.r_{+}^{2} \int_{0}^{1} d u^{\prime} \frac{\rho_{0}^{2}\left(u^{\prime}\right)}{u^{\prime 2}} \partial_{u} \int d \mathbf{x}^{\prime} G_{s}\left(u, u^{\prime} \mid \mathbf{x}-\mathbf{x}^{\prime}\right) \sigma\left(\mathbf{x}^{\prime}\right)\right|_{u \rightarrow 0} .
$$

One can see that the dependence on the dark matter sector (dependence on $\tilde{\alpha}$ ) cancels in the first term and the current in the presence of the dark matter sector is provided by

$$
<J_{a}>^{\mathrm{DM}}=<J_{a}>+\beta_{a}(\alpha) .
$$

From the above relation it can be concluded that if we take an integration constants $c_{i}$ equal to zero, one receives that the dark matter sector current is the same as in the 'ordinary' case.

\section{Free energy}

In this section we shall find free energy of the considered system, i.e., Maxwell dark matter sector vortex configuration in $(2+1)$-dimensions. The free energy notion is important from the point of view of the thermodynamical stability of the aforementioned configuration. It 
turns out that in holographic approach to the physical problems the free energy for the boundary theory can be determined as the on-shell gravity action in the bulk.

In our case the on-shell action bounded with the scalar field in question vanishes identically. It is caused by the fact that the scalar field has a compact support and satisfies the boundary conditions of the form $\psi \sim c_{1} u^{2}$, where $c_{1}$ is constant, at the AdS boundary. By virtue of the above one can restrict the attention to Maxwell and dark matter U(1)gauge fields.

$$
S_{\text {on-shell }}=-\int_{M} d^{4} x \sqrt{-g}\left(\frac{1}{4} F_{\mu \nu} F^{\mu \nu}+\frac{\alpha}{4} F_{\mu \nu} B^{\mu \nu}\right) .
$$

Let us expand the above action in terms of $\epsilon_{H}$

$$
S_{\text {on-shell }}=S^{(0)}+\epsilon_{H} S^{(1)}+\epsilon_{H}^{2} S^{(2)}+\ldots
$$

On the other hand, for the gauge fields one obtains

$$
F_{\mu \nu}^{(i)}=\partial_{\mu} A_{\nu}^{(i)}-\partial_{\nu} A_{\mu}^{(i)}, \quad B_{\mu \nu}^{(i)}=\partial_{\mu} B_{\nu}^{(i)}-\partial_{\nu} B_{\mu}^{(i)}, \quad i=0,1,2, \ldots
$$

The $S^{(0)}$ coefficient implies

$$
-S^{(0)}=\int_{\mathcal{M}} d^{4} x \sqrt{-g}\left(\frac{1}{4} F_{\mu \nu}^{(0)} F^{\mu \nu(0)}+\frac{\alpha}{4} F_{\mu \nu}^{(0)} B^{\mu \nu(0)}\right) .
$$

The first term on the right-hand side of (4.4) accords to a trivial configuration without any scalar field, i.e., $\psi=0$. However, the other terms in the expansion described by the relation (4.2) possess the information about states in which one has non-zero condensate.

Let us consider the $S^{(1)}$ coefficient which may be rewritten in the form as

$$
\begin{aligned}
-S^{(1)} & =\int_{\mathcal{M}} d^{4} x \sqrt{-g}\left[\frac{1}{2} F_{\mu \nu}^{(1)} F^{\mu \nu(0)}+\frac{\alpha}{4}\left(F_{\mu \nu}^{(1)} B^{\mu \nu(0)}+F_{\mu \nu}^{(0)} B^{\mu \nu(1)}\right)\right] \\
& =\left.\int_{\partial \mathcal{M}} d \Sigma_{u} F^{u \nu(0)} A_{\nu}^{(1)}\right|_{u \rightarrow 0}+\frac{\alpha}{2}\left[\int_{\partial \mathcal{M}} d \Sigma_{u}\left(B^{u \nu(0)} A_{\nu}^{(1)}+F^{u \nu(0)} B_{\nu}^{(1)}\right)\right]_{u \rightarrow 0},
\end{aligned}
$$

where we used the equations of motion for the zeroth order, i.e., $\nabla_{\mu} F^{\mu \nu(0)}=0$ which in turn implies that $\nabla_{\mu} B^{\mu \nu(0)}=0$. These terms vanish because of the fact that one has to take into account the boundary theory at some fixed value of the chemical potential $\mu$.

The $S^{(2)}$ coefficient is provided by the following relations:

$$
\begin{aligned}
-S^{(2)}= & \int_{\mathcal{M}} d^{4} x \sqrt{-g}\left[\frac{1}{2} F_{\mu \nu}^{(1)} F^{\mu \nu(0)}+\frac{1}{4} F_{\mu \nu}^{(1)} F^{\mu \nu(1)}\right. \\
& \left.+\frac{\alpha}{4}\left(F_{\mu \nu}^{(2)} B^{\mu \nu(0)}+F_{\mu \nu}^{(1)} B^{\mu \nu(1)}+F_{\mu \nu}^{(0)} B^{\mu \nu(2)}\right)\right] \\
= & \left.\int_{\partial \mathcal{M}} d \Sigma_{u}\left(F^{u \nu(0)} A_{\nu}^{(2)}+\frac{1}{2} F^{u \nu(1)} A_{\nu}^{(1)}\right)\right|_{u \rightarrow 0}-\frac{3}{2} \int_{\mathcal{M}} d^{4} x \sqrt{-g} \frac{j^{\nu(1)}}{\tilde{\alpha}} A_{\nu}^{(1)} \\
& +\left.\int_{\partial \mathcal{M}} d \Sigma_{u} \frac{\alpha}{2}\left(B^{u \nu(0)} A_{\nu}^{(2)}+F^{u \nu(0)} B_{\nu}^{(2)}+F_{\mu \nu}^{(1)} B^{\mu \nu(1)}\right)\right|_{u \rightarrow 0},
\end{aligned}
$$


where one has used the equations of motion for the first order, i.e., $\tilde{\alpha} \nabla_{\mu} F^{\mu \nu(1)}=j^{\nu(1)}$. The 'orthogonality condition' [53]

$$
\int_{\mathcal{M}} d^{4} x \sqrt{-g} A_{\mu}^{(1)} j^{\mu(1)}=0
$$

can be implemented and the second term in the third line vanishes. Consequently we arrive at the following expression:

$$
-S_{\text {on-shell }}=\int_{\partial \mathcal{M}} d \Sigma_{u}\left[\frac{\alpha}{2}\left(F^{u \nu(0)} B_{\nu}+B^{u \nu(0)} A_{\nu}\right)+\frac{\epsilon^{2}}{2} A_{\nu}^{(1)}\left(F^{u \nu(1)}+\alpha B^{u \nu(1)}\right)\right]+\mathcal{O}\left(\epsilon_{H}^{3}\right) .
$$

The same arguments as applied in $S^{(1)}$ analysis, lead us to the conclusion that the first term on the right-hand side of equation (4.8) vanishes. In the next step we use the fact that $A_{t}^{(1)}(\mathbf{x}, 0)=0$. Consequently, by virtue of the above the on-shell action is provided by the relation of the form

$$
-S_{\text {on-shell }}=\left.\frac{\epsilon^{2}}{2} \int d^{3} x \delta^{a b}\left(F_{u a}^{(1)}+\alpha B_{u a}^{(1)}\right) A_{b}^{(1)}\right|_{u \rightarrow 0}+\mathcal{O}\left(\epsilon_{H}^{3}\right) .
$$

Having in mind the relation for $\left\langle J_{a}>^{\mathrm{DM}}\right.$ one has that

$$
-S_{\text {on-shell }}=\frac{\epsilon^{2}}{2} \int d^{3} x \delta^{a b}<J_{a}>\left.^{\mathrm{DM}} A_{b}^{(1)}\right|_{u \rightarrow 0}+\mathcal{O}\left(\epsilon_{H}^{3}\right) .
$$

Putting together all those results, we obtain that the expression describing free energy for the considered theory with dark matter sector in $(2+1)$-dimensions. It yields

$$
\begin{aligned}
F=-\frac{\epsilon^{2}}{2} \int_{\mathcal{R}^{2}} d^{2} \mathbf{x} \delta^{a b}<J_{a}>\left.^{\mathrm{DM}} A_{b}^{(1)}\right|_{u \rightarrow 0}= & -\frac{\epsilon^{2}}{2} B_{c_{2}} \int_{\mathcal{R}^{2}} d^{2} \mathbf{x} \Theta(\mathbf{x}) \\
& -\frac{\epsilon^{2}}{2} \int_{\mathcal{R}^{2}} d^{2} \mathbf{x} \beta_{i}(\alpha) A^{i(1)},
\end{aligned}
$$

where $B_{c_{2}}$ stands for 'the upper magnetic' U(1)-gauge Maxwell field.

Let us suppose that we take into account some bounded region $V$ of the two-dimensional hypersurface $\mathcal{R}^{2}$ in question. The free energy density may be written as

$$
\mathcal{F}=\frac{F}{V}=-\frac{\epsilon^{2}}{2} B_{c_{2}}<\Theta(\mathbf{x})>-\frac{\epsilon^{2}}{2}<\beta_{i}(\alpha) A^{i(1)}>,
$$

where $<\Theta(\mathbf{x})>$ and $<\beta_{i}(\alpha) A^{i(1)}>$ denote the averages of $\Theta(\mathbf{x})$ and $\beta_{i}(\alpha) A^{i(1)}$ over the two-dimensional hypersurface $\mathcal{R}^{2}$.

It is worth pointing out that the free energy density consists of two terms, first one is connected with the ordinary Maxwell field while the other one is bounded with dark matter sector. Because of the fact that $\mathcal{F}$ is negative one can conclude that the considered system is more stable over the trivial configuration where there is no charge condensate, i.e., the value of the scalar field $\psi$ is equal to zero. Moreover, the free energy is smaller for the dark matter sector, which means that the vortices create more easily in the presence of dark matter than without it. Perhaps this could be the answer why the cosmic web of tendrils (resembling vortices in the large cosmological scales) form first and then an ordinary matter condenses on them to form galaxies and clusters of galaxies. 


\section{$5 \quad$ Superconducting coherence length}

We turn now to the analysis of the superconducting length and the influence of the dark matter sector on it. In our analysis we shall consider the following components of the Maxwell and scalar fields:

$$
A_{\mu}=\left(\phi(u), 0,0, A_{y}\right), \quad \psi=\psi(u) .
$$

By virtue of the above choice we can straightforwardly verify that the equations of motion imply

$$
\begin{aligned}
\partial_{u}^{2} \phi(u)-\frac{2 r_{+} \psi^{2} \phi}{\tilde{\alpha} u^{4} f(u)} & =0, \\
\partial_{u}\left(f(u) \partial_{u} \psi\right)-\frac{m^{2} r_{+}^{2}}{u^{4}} \psi+\frac{\phi^{2} r_{+}^{2}}{f(u) u^{4}} \psi-\frac{1}{u^{4}} A_{y}^{2} \psi & =0 .
\end{aligned}
$$

It happens that the superconducting coherence length $\xi$ can be connected with the correlation length of the order parameter in the momentum space [48]. $\chi$ emerges as the pole of the static correlation function of the order parameter in the Fourier space. Namely, one can write that

$$
<\mathcal{O}(k) \mathcal{O}(-k)>\sim \frac{1}{|k|^{2}+\frac{1}{\xi^{2}}} .
$$

As in [48] the pole of the static correlation function of a dual field operator can be found by solving the eigenvalue problem for the static perturbation subject to the condition that the wave number $k$ of the corresponding bulk field is given by $1 / \xi^{2}=-k_{*}{ }^{2}$, where $k_{*}$ is a wave number allowed as eigenvalues [48]. The aforementioned condition gives us the pole of the static correlation function. Because of the fact that near the critical point, when $T \rightarrow T_{c}$, the coherence length is divergent, one ought to solve the eigenvalue problem being subject to the condition

$$
\lim _{\epsilon_{T} \rightarrow 0}\left(-k^{2}\right)=-k_{*}^{2}=\frac{1}{\xi^{2}}=0,
$$

where $\epsilon_{T}=\left(T_{c}-T\right) / T_{c}$ with the auxiliary demand that $\left|\epsilon_{T}\right| \ll 1$. In order to solve the equations of motion let us perform the perturbative analysis in the fluctuations of the fields under considerations given by

$$
\begin{aligned}
& \phi=\varphi(u)+\epsilon_{T} A_{t}^{(1)}(u)+\mathcal{O}\left(\epsilon_{T}^{2}\right), \\
& \psi=\sqrt{\epsilon_{T}} \psi_{1}(u)+\epsilon_{T}^{\frac{3}{2}} \psi_{2}(u)+\mathcal{O}\left(\epsilon_{T}^{2}\right) .
\end{aligned}
$$

In the leading order of the $\epsilon_{T}$ parameter, the underlying equations of motion can be cast in the forms as stated below. Namely, in the first order of $\epsilon_{T}$-order we get

$$
\partial_{u}^{2} A_{t}^{(1)}(u)-\frac{2 r_{+}^{2}}{\tilde{\alpha} u^{4} f(u)} \psi_{1}^{2}(u) \varphi(u)=0 .
$$

On the other hand, at $\sqrt{\epsilon_{T}}$-order they imply

$$
\partial_{u}\left(f(u) \partial_{u} \psi_{1}(u)\right)-\frac{m^{2} r_{+}^{2}}{u^{4}} \psi_{1}(u)+\frac{r_{+}^{2} \varphi^{2}(u) \psi_{1}(u)}{f(u) u^{4}}-\frac{1}{u^{4}} A_{y}^{2} \psi_{1}(u)=0,
$$


and at $\epsilon_{T}^{\frac{3}{2}}$-order, one arrives at the relation as follows:

$$
\partial_{u}\left(f(u) \partial_{u} \psi_{2}(u)\right)-\frac{m^{2} r_{+}^{2}}{u^{4}} \psi_{2}(u)+\frac{2 r_{+}^{2} \varphi(u) \psi_{1}(u) A_{t}^{(1)}(u)}{f(u) u^{4}}=0 .
$$

In order to determine the coherence length, we shall elaborate fluctuations around the background fields $A_{\mu}(u)$ and $\psi(u)$. It turned out that static perturbations will be adequate for our purpose. Thus, we pay attention to the fluctuations with only one spatial direction along $x$-direction. The linear perturbations of gauge fields and scalar one, will yield

$$
\begin{aligned}
\delta A_{\mu} & =\left[a_{t}(u, k) d t+a_{x}(u, k) d x+a_{y}(u, k) d y\right] e^{i k x}, \\
\delta \psi & =[b(u, k)+i \bar{b}(u, k)] e^{i k x} .
\end{aligned}
$$

The boundary conditions for the gauge and scalar fields should be imposed. Namely, let us suppose that at the black brane horizon $(u=1)$ and near of the AdS spacetime boundary $(u=0)$, the asymptotic behavior of the fields in question imply

$$
\begin{aligned}
\left.a_{t}(u, k)\right|_{u \rightarrow 1} & =0, \\
\left.b(u, k)\right|_{u \rightarrow 1} & =\text { regular, } \\
\left.a_{t}(u, k)\right|_{u \rightarrow 0} & =\text { const } u, \\
\left.b(u, k)\right|_{u \rightarrow 0} & =\text { const } u^{2} .
\end{aligned}
$$

Intersecting (5.11)-(5.12) into the underlying equations of motion provides the following:

$$
\begin{aligned}
k^{2} a_{t}=f(u) u^{2} \partial_{u}^{2} a_{t}-\frac{2 \psi^{2} r_{+}^{2} a_{t}}{\tilde{\alpha} u^{2}}-\frac{4 \psi b r_{+}}{\tilde{\alpha} u^{2}} \phi & =0, \\
k^{2} a_{y}=\partial_{u}\left[u^{2} f(u) \partial_{u} a_{y}\right]-\frac{2 \psi^{2} r_{+}^{2} a_{y}}{\tilde{\alpha} u^{2}}-\frac{4 \psi b A_{y} r_{+}^{2}}{\tilde{\alpha} u^{2}} & =0, \\
k^{2} b=u^{2} \partial_{u}\left[f(u) \partial_{u} b\right]+\frac{r_{+}^{2} b \phi^{2}}{f(u) u^{2}}-A_{y}^{2} b-\frac{m^{2} r_{+}^{2} b}{u^{2}} & \\
+\frac{2 a_{t} \phi \psi r_{+}^{2}}{f(u) u^{2}}-2 a_{t} A_{y} \psi & =0, \\
\partial_{u}\left[u^{2} f(u) \partial_{u} a_{x}\right]-\frac{2 r_{+}^{2}}{\tilde{\alpha} u^{2}} \psi^{2} a_{x} & =0 .
\end{aligned}
$$

The Wick rotation of the wave vector $k, k \rightarrow i k$ and substitution the relation (5.6) reveal the following:

$$
\begin{gathered}
-k^{2} a_{t}=f(u) u^{2} \partial_{u}^{2} a_{t}-\frac{2 \epsilon_{T} \psi_{1}^{2} r_{+}^{2} a_{t}}{\tilde{\alpha} u^{2}}-\frac{4 \sqrt{\epsilon_{T}} \psi_{1} b r_{+} \varphi}{\tilde{\alpha} u^{2}} \phi=0, \\
-k^{2} b=\left(D_{b}+\frac{2 \epsilon_{T} r_{+}^{2} \varphi A_{t}^{(1)}(u)}{f(u) u^{2}}\right) b+\frac{2 \sqrt{\epsilon_{T}} a_{t} \varphi \psi_{1} r_{+}^{2}}{f(u) u^{2}} \\
-2 \sqrt{\epsilon_{T}} a_{y} A_{y} \psi_{1}=0,
\end{gathered}
$$


where we have define the differential operator $D_{b}$ by the relation

$$
D_{b}=u^{2} \partial_{u}\left(f(u) \partial_{u}\right)+\frac{r_{+}^{2} \varphi^{2}}{f(u) u^{2}}-A_{y}^{2}-\frac{m^{2} r_{+}^{2}}{u^{2}} .
$$

The zeroth order solutions of the set of equations (5.21)-(5.22), which are consistent with the asymptotical behavior near the black brane event horizon and AdS spacetime boundary, have the forms given by the relations

$$
b^{(0)}=\psi_{1}, \quad a_{t}^{(0)}=0, \quad a_{y}^{(0)}=0 .
$$

The zeroth order solutions are in accord with the fact that at this order they are only functions of $u$-coordinate. Having in mind that perturbations described by the equations (5.11)-(5.12) have the spatial dependence on $x$-direction, we conclude that the first non-trivial corrections emerges at $\sqrt{\epsilon_{T}}$-order. Thus, we suppose that

$$
a_{t}=\sqrt{\epsilon_{T}} \tilde{a}_{t}, \quad a_{y}=\sqrt{\epsilon_{T}} \tilde{a}_{y}
$$

and substitute the above expressions into the relations for $a_{t}, a_{y}, b$. Consequently, one obtains

$$
\begin{aligned}
& -k^{2} \tilde{a}_{t}=f(u) u^{2} \partial_{u}^{2} \tilde{a}_{t}-\frac{4 \psi_{1} b r_{+}^{2} \varphi}{\tilde{\alpha} u^{2}}-2 \epsilon_{T} \frac{\psi_{1}^{2} r_{+}^{2}}{\tilde{\alpha} u^{2}} \tilde{a}_{t}=0, \\
& -k^{2} b=D_{b} b+\epsilon_{T}\left(\frac{2 r_{+}^{2} \varphi A_{t}^{(1)}(u) b}{f(u) u^{2}}+\frac{2 \tilde{a}_{t} \varphi \psi_{1} r_{+}^{2}}{f(u) u^{2}}-2 \tilde{a}_{y} A_{y} \psi_{1}\right)=0, \\
& -k^{2} \tilde{a}_{y}=\partial_{u}\left(u^{2} f(u) \partial_{u} \tilde{a}_{y}\right)-\frac{4 \psi_{1}^{2} A_{y} r_{+}^{2}}{\tilde{\alpha} u^{2}}+\mathcal{O}\left(\epsilon_{T}^{n \geq 1 / 2}\right) .
\end{aligned}
$$

Moreover we write down the set of expansions for the coefficients of the static perturbations

$$
\begin{aligned}
b & =\psi_{1}+\epsilon_{T} b^{(1)}+\mathcal{O}\left(\epsilon_{T}^{2}\right), \\
\tilde{a}_{t} & =\tilde{a}_{t}^{(0)}+\mathcal{O}\left(\epsilon_{T}\right), \\
\tilde{a}_{y} & =\tilde{a}_{y}^{(0)}+\mathcal{O}\left(\epsilon_{T}\right), \\
k_{*}^{2} & =\epsilon_{T} k_{1}^{2}+\mathcal{O}\left(\epsilon_{T}^{2}\right) .
\end{aligned}
$$

By virtue of the equations (5.26)-(5.27) as well as (5.8)-(5.9), taking into account the limit $\epsilon_{T} \rightarrow 0$, which means that $k^{2} \rightarrow k_{*}^{2}$, we can readily verify that

$$
\begin{aligned}
\partial_{u}^{2} \tilde{a}_{t}^{(0)}\left(u, k_{*}\right)= & \frac{4 \psi^{2} r_{+}^{2} \varphi}{\tilde{\alpha} f(u) u^{4}}=2 \partial_{u}^{2} A_{t}^{(1)}(u), \\
-k_{1}^{2} \psi_{1}= & D_{b} b^{(1)}\left(u, k_{*}\right) \\
& +\left(\frac{2 r_{+}^{2} \psi_{1} \varphi A_{t}^{(1)}(u)}{f(u) u^{2}}+\frac{2 \tilde{a}_{t}^{(0)} \varphi \psi_{1} r_{+}^{2}}{f(u) u^{2}}-2 \tilde{a}_{y}^{(0)} A_{y} \psi_{1}\right)=0 .
\end{aligned}
$$

In order to calculate the superconducting coherence length we define the scalar product defined by

$$
<\psi_{1} \mid \psi_{1}>=\int_{0}^{1} d u \frac{\psi_{1}^{*} \psi_{1}}{u^{2}}
$$


The form of the scalar product reveals the fact that the operator $D_{b}$ is the Hermitian one. In the next step, we multiply the equation for $\tilde{k}_{1}$ by the bra $<\psi_{1} \mid$. On this account it is customary to write

$$
\begin{aligned}
-k_{1}^{2}<\psi_{1} \mid \psi_{1}>= & <\psi_{1}\left|D_{b} b^{(1)}>+<\psi_{1}\right| \frac{2 r_{+}^{2} \psi_{1} \varphi A_{t}^{(1)}(u)}{f(u) u^{2}}> \\
& -\frac{\tilde{\alpha}}{2} \int_{0}^{1} d u\left(\frac{d \tilde{a}_{t}^{(0)}}{d u}\right)^{2}-2 A_{y} \int_{0}^{1} \frac{d u}{u^{2}} \psi_{1}^{2} \tilde{a}_{y}^{(0)} .
\end{aligned}
$$

The first term on the right-hand side can be found considering $\epsilon_{T}^{3 / 2}$-order behavior of the equations of motion for the underlying theory. Namely the relation (5.10) enables us to write

$$
D_{b} \psi_{2}=-\frac{2 r_{+}^{2} \psi_{1} \varphi A_{t}^{(1)}(u)}{f(u) u^{2}},
$$

which in turn leads us to the conclusion that $\left\langle\psi_{1} \mid D_{b} \psi_{2}\right\rangle=0$.

Finally, coming back to the original vector wave, by performing the Wick rotation as well as taking into account the limit when $\epsilon_{T} \rightarrow 0$, we receive the searched for expression

$$
-k_{1}^{2}=\frac{\mathcal{P}}{\mathcal{M}} \text {. }
$$

For the brevity of the notation we have defined the above quantities $\mathcal{P}$ and $\mathcal{M}$ as

$$
\begin{aligned}
\mathcal{P} & =\frac{\tilde{\alpha}}{2} \int_{0}^{1} d u\left(\frac{d \tilde{a}_{t}^{(0)}}{d u}\right)^{2}+2 A_{y} \int_{0}^{1} d u \psi_{1}^{2} \tilde{a}_{y}^{(0)}, \\
\mathcal{M} & =\int_{0}^{1} d u \frac{\psi_{1}^{*} \psi_{1}}{u^{2}} .
\end{aligned}
$$

On this account the superconducting coherence length in the theory with dark matter sector yields

$$
\xi=\sqrt{\frac{\mathcal{M}}{\mathcal{P}}}\left(1-\frac{T}{T_{c}}\right)^{\frac{1}{2}} \sim \tilde{\alpha}^{-\frac{1}{2}}\left(1-\frac{T}{T_{c}}\right)^{\frac{1}{2}} .
$$

The coherence length depends on the $\alpha$-coupling constant (which in turn describes the dependence on the dark matter model). The main conclusion of these calculations is that the smaller $\alpha$ we consider, the greater value of the superconducting length we obtain.

\section{London equation with dark matter sector}

This section is devoted to calculations of the magnetic penetration depth and the number density of the superfluid for Maxwell dark sector vortices in $(2+1)$-dimensions in the presence of a homogeneous external magnetic field. The magnetic field is chosen as perpendicular to the two-dimensional hypersurface. Let us introduce the ansatz of the form as

$$
\delta A_{y}(u, x)=a_{y}(u) x
$$

which is equivalent to the perturbation of the gauge field with the wave vector $k=0$. 
As was mentioned in section $2, A_{y}$ can be connected with the dark matter magnetic field or Maxwell magnetic field. For generality of our considerations we set in what follows $A_{y}$. Inserting it into equation (5.18) one readily writes down

$$
\partial_{u}\left(u^{2} f(u) \partial_{u} a_{y}\right)-\frac{2 r_{+}^{2} \psi^{2} a_{y}}{\tilde{\alpha} u^{2}}-\frac{4 \psi b A_{y} r_{+}^{2}}{\tilde{\alpha} u^{2}}=0
$$

As in the previous section we assume the following perturbative expansion:

$$
\begin{aligned}
\psi & =\sqrt{\epsilon_{T}} \psi_{1}(u)+\epsilon_{T}^{3 / 2} \psi_{2}(u)+\mathcal{O}\left(\epsilon_{T}^{5 / 2}\right) \\
a_{y} & =a_{y}^{(0)}+\epsilon_{T} a_{y}^{(1)}+\mathcal{O}\left(\epsilon_{T}^{3}\right) \\
b & =\psi_{1}(u)+\sqrt{\epsilon_{T}} b^{(1)}+\mathcal{O}\left(\epsilon_{T}\right) .
\end{aligned}
$$

Up to $\epsilon_{T}^{0}$ and to $\epsilon_{T}^{1}$ orders the equations are given respectively by

$$
\begin{aligned}
\partial_{u}\left(u^{2} f(u) \partial_{u} a_{y}^{(0)}\right) & =0, \\
\partial_{u}\left(u^{2} f(u) \partial_{u} a_{y}^{(1)}\right)-\frac{2 r_{+}^{2}}{\tilde{\alpha} u^{2}} \psi_{1}^{2}(u) a_{y}^{(0)}-\frac{4 b^{(1)} \psi_{1}(u) r_{+}^{2}}{\tilde{\alpha} u^{2}} A_{y} & =0 .
\end{aligned}
$$

From the equation (6.6) it can be seen that $a_{y}^{(0)}$ is constant and $a_{y}^{(1)}$ solution can be formally written as

$$
\begin{aligned}
& a_{y}^{(0)}=C_{0}=\text { const } \\
& a_{y}^{(1)}=C_{1}-2 C_{0} \int_{0}^{u} \frac{d u^{\prime \prime}}{1-u^{\prime \prime 3}} \int_{u^{\prime \prime}}^{1} \frac{d u^{\prime}}{\tilde{\alpha} u^{\prime 2}} \psi_{1}\left(u^{\prime}\right)\left(\psi_{1}\left(u^{\prime}\right)+\frac{2 b^{(1)}\left(u^{\prime}\right) A_{y}}{C_{0}}\right)+\mathcal{O}\left(\epsilon_{T}^{2}\right)
\end{aligned}
$$

To proceed further, we set $C_{1}=0$ and $C_{0}=A_{y}$. On this account the relation (6.1) for $\delta A_{y}(u, x)$ implies

$$
\delta A_{y}(u, x) \simeq \delta A_{y}^{(0)}(x)\left(1-2 \epsilon_{T} u \int_{u^{\prime \prime}}^{1} \frac{d u^{\prime}}{\tilde{\alpha} u^{\prime 2}} \psi_{1}\left(u^{\prime}\right)\left(\psi_{1}\left(u^{\prime}\right)+2 b^{(1)}\left(u^{\prime}\right)\right)\right)+\mathcal{O}\left(\epsilon_{T}^{2}\right),
$$

where $\delta A_{y}^{(0)}=\lim _{u \rightarrow 0} \delta A_{y}(U, x)=A_{y}$.

On the other hand, from the asymptotic behavior of $\delta A_{y}$ near the AdS spacetime boundary

$$
\delta A_{y}=\delta A_{y}^{(0)}+\frac{\delta A_{y}^{(1)}}{r}+\ldots,
$$

and taking into account the definition $r_{+}=4 \pi T_{c} / 3$, we can read off the form of $\left\langle J_{y}(x)\right\rangle$ when $T \rightarrow T_{c}$. Namely, it is given by the relation

$$
<J_{y}(x)>_{u \rightarrow 0}=-\frac{8 \pi \epsilon_{T}}{3} T_{c} \delta A_{y}^{(0)}(x) \int_{0}^{1} \frac{d u}{\tilde{\alpha} u^{2}} \mathcal{K}(u, k)+\mathcal{O}\left(\epsilon_{T}^{2}\right),
$$

where we have denoted by $\mathcal{K}(u, k)$ the relation provided by

$$
\mathcal{K}(u, k)=\psi_{1}^{2}(u)+2 \psi_{1}(u) b^{(1)} .
$$


Let us find the behavior of the integral (6.12) near the AdS spacetime boundary. Near the boundary (for $m^{2}=-2$ ) the scalar field $\psi_{1}$ is proportional to the condensation operator $<\mathcal{O}>$. Because of the fact that we reach almost the critical temperature, the condensation operator plays the role of the order parameter for the boundary theory. In general, the considered scalar field solution can be expressed as a function of the $u$-coordinate [48] in the form as

$$
\psi(u)=\psi^{m} f(u),
$$

where $f(u)$ is the solution of equation satisfying the $\operatorname{limit}_{\lim _{u \rightarrow 0}} f(u)=u^{\Delta_{m}}$, and regularity condition at the black hole event horizon. By virtue of this, we set

$$
\psi_{1} \sim<\mathcal{O}>u^{2} .
$$

For the upper limit in the considered integral, i.e., for $u=1, \psi_{1}$ is perfectly regular. All these facts suggest that the integral in question is well defined and has the finite value.

As was revealed in [53] the form of $\left\langle J_{y}\right\rangle$ closely resembles the expression from the Ginzburg-Landau theory, so-called London equation, in which the order parameter $\psi$ is coupled to the $\mathrm{U}(1)$-gauge field $A_{a}$ and the current $J_{a}$ is provided by

$$
J_{a}=-\frac{e^{2}}{m} \psi^{2} A_{a}=-e n_{s} A_{a}
$$

where $e$ and $m$ are effective charge and mass of the order parameter. $n_{s}$ is connected with the superfluid number density. In the expression for the average spatial current in y-direction, $\delta A_{y}^{(0)}$ plays the role of an external source. On the contrary, in London equation $A_{a}$ is built from the spatial average of the microscopic field as well as an external field. In the considered attitude we have no dynamical photon (the current does not produce its own magnetic field). Summing it all up, it means that the external U(1)-gauge field $\delta A_{y}^{(0)}$ equals to the macroscopic gauge field in the AdS/CFT attitude. So the comparison of $J_{a}$ and $\left\langle J_{y}>\right.$ reveals that, near the boundary of the AdS spacetime we have that the number density of the superfluid particles is given by

$$
n_{s}^{\mathrm{DM}}=\frac{8 \pi T_{c}}{3 \tilde{\alpha}}<\mathcal{O}>^{2} F(u, k)=\frac{n_{s}}{\tilde{\alpha}},
$$

where $F(u, k)$ stands for the integral

$$
F(u, k)=\int_{0}^{1} \frac{d u}{u^{2}} \mathcal{K}(u, k) .
$$

On the other hand, the magnetic penetration depth implies

$$
\lambda^{\mathrm{DM}}=\sqrt{\tilde{\alpha}} \lambda .
$$

Inspection of the equations (6.17) and (6.19) reveals that the bigger $\alpha$-coupling constant one takes the greater $n_{s}^{\mathrm{DM}}$ we get. On the contrary, the smaller $\alpha$ the greater magnetic penetration we have.

It is interesting to note that dark matter affects both the penetration depth and coherence length in the same way leaving their ratio intact $\lambda^{\mathrm{DM}} / \xi^{\mathrm{DM}}=\lambda / \xi$. Noting that this ratio decides if the superconductor is of first or second type, the conclusion is that, at least in the probe limit, the dark matter does not affect classification of the holographic superconductors. 


\section{Conclusions}

In the paper we have considered AdS/CFT-gauge/gravity, correspondence in order to study linear fluctuations of scalar field solution in the s-wave holographic superconductor under the assumption of the probe limit, i.e., the fluctuations do not backreact on the gravitational field. We have analyzed the theory in which U(1)-gauge Maxwell field is coupled with the other gauge field which mimics the presence of the dark matter sector.

The main aim of the work was to answer the question how dark matter sector ( $\alpha$ coupling constant of these aforementioned gauge fields) will modify characteristics of vortex solutions, like the coherence length, superfluid density and magnetic penetration depth. It happened that both, the coherence length and the penetration depth were affected by the presence of the dark matter sector in the same way. The smaller value of $\alpha$ is taken into account the greater value of the $\xi$ and $\lambda$ is obtained. On the other hand, the smaller $\alpha$ we have, the greater superfluid density we get.

We have also found that the free energy for the vortex configuration turns out to be negative and for the configuration with dark matter sector lesser than in the 'ordinary' Maxwell case. This fact enables us to conclude that the vortex configurations are stable over the trivial configuration where there is no charge scalar condensate. Secondly, because of the fact that in the presence of dark matter the vortices build less free energy configuration than ordinary matter ones (e.g., Maxwell or Chern-Simons), it can give rise to the answer why in the Early Universe first the web of dark matter formed and next on these tendrils the ordinary matter condensed forming cluster of galaxies.

\section{Acknowledgments}

MR was partially supported by the grant of the National Science Center DEC-2014/15/B/ ST2/00089 and KIW by the grant DEC-2014/13/B/ST3/04451.

Open Access. This article is distributed under the terms of the Creative Commons Attribution License (CC-BY 4.0), which permits any use, distribution and reproduction in any medium, provided the original author(s) and source are credited.

\section{References}

[1] J.M. Maldacena, The large- $N$ limit of superconformal field theories and supergravity, Int. J. Theor. Phys. 38 (1999) 1113 [hep-th/9711200] [INSPIRE].

[2] E. Witten, Anti-de Sitter space and holography, Adv. Theor. Math. Phys. 2 (1998) 253 [hep-th/9802150] [INSPIRE].

[3] S.S. Gubser, I.R. Klebanov and A.M. Polyakov, Gauge theory correlators from noncritical string theory, Phys. Lett. B 428 (1998) 105 [hep-th/9802109] [INSPIRE].

[4] J.P. Gauntlett, J. Sonner and T. Wiseman, Holographic superconductivity in M-theory, Phys. Rev. Lett. 103 (2009) 151601 [arXiv:0907.3796] [INSPIRE].

[5] S. Sachdev, What can gauge-gravity duality teach us about condensed matter physics?, Ann. Rev. Condensed Matter Phys. 3 (2012) 9 [arXiv:1108.1197] [InSPIRE]. 
[6] A.G. Green, An Introduction to Gauge Gravity Duality and Its Application in Condensed Matter, Contemp. Phys. 54 (2013) 33 [arXiv:1304.5908] [InSPIRE].

[7] S.A. Hartnoll, C.P. Herzog and G.T. Horowitz, Building a Holographic Superconductor, Phys. Rev. Lett. 101 (2008) 031601 [arXiv:0803.3295] [inSPIRE].

[8] J.-W. Chen, Y.-J. Kao, D. Maity, W.-Y. Wen and C.-P. Yeh, Towards A Holographic Model of D-Wave Superconductors, Phys. Rev. D 81 (2010) 106008 [arXiv:1003.2991] [INSPIRE].

[9] F. Benini, C.P. Herzog, R. Rahman and A. Yarom, Gauge gravity duality for d-wave superconductors: prospects and challenges, JHEP 11 (2010) 137 [arXiv:1007.1981] [INSPIRE].

[10] M. Rogatko and K.I. Wysokiński, Remarks on the Hall conductivity in chiral superconductors: weak vs. strong coupling approach, Acta Phys. Polon. A 126 (2014) A9.

[11] H.-B. Zeng, Z.-Y. Fan and H.-S. Zong, d-wave Holographic Superconductor Vortex Lattice and Non-Abelian Holographic Superconductor Droplet, Phys. Rev. D 82 (2010) 126008 [arXiv: 1007.4151] [INSPIRE].

[12] S.S. Gubser and S.S. Pufu, The gravity dual of a p-wave superconductor, JHEP 11 (2008) 033 [arXiv:0805.2960] [INSPIRE].

[13] G.T. Horowitz and B. Way, Complete Phase Diagrams for a Holographic Superconductor/Insulator System, JHEP 11 (2010) 011 [arXiv:1007.3714] [INSPIRE].

[14] P. Basu, J. He, A. Mukherjee and H.-H. Shieh, Hard-gapped Holographic Superconductors, Phys. Lett. B 689 (2010) 45 [arXiv:0911.4999] [inSPIRE].

[15] F. Aprile, D. Rodriguez-Gomez and J.G. Russo, p-wave Holographic Superconductors and five-dimensional gauged Supergravity, JHEP 01 (2011) 056 [arXiv:1011.2172] [INSPIRE].

[16] S. Gangopadhyay and D. Roychowdhury, Analytic study of properties of holographic p-wave superconductors, JHEP 08 (2012) 104 [arXiv:1207.5605] [INSPIRE].

[17] M. Ammon, J. Erdmenger, V. Grass, P. Kerner and A. O'Bannon, On Holographic p-wave Superfluids with Back-reaction, Phys. Lett. B 686 (2010) 192 [arXiv:0912.3515] [INSPIRE].

[18] S. Liu and Y.-Q. Wang, Holographic model of hybrid and coexisting s-wave and p-wave Josephson junction, Eur. Phys. J. C 75 (2015) 493 [arXiv:1504.06918] [InSPIRE].

[19] R.-G. Cai, L. Li, H.-Q. Zhang and Y.-L. Zhang, Magnetic Field Effect on the Phase Transition in AdS Soliton Spacetime, Phys. Rev. D 84 (2011) 126008 [arXiv:1109.5885] [INSPIRE].

[20] R.-G. Cai, H.-F. Li and H.-Q. Zhang, Analytical Studies on Holographic Insulator/Superconductor Phase Transitions, Phys. Rev. D 83 (2011) 126007 [arXiv: 1103.5568] [INSPIRE].

[21] A. Akhavan and M. Alishahiha, P-Wave Holographic Insulator/Superconductor Phase Transition, Phys. Rev. D 83 (2011) 086003 [arXiv:1011.6158] [InSPIRE].

[22] A. Amoretti, A. Braggio, N. Maggiore, N. Magnoli and D. Musso, Coexistence of two vector order parameters: a holographic model for ferromagnetic superconductivity, JHEP 01 (2014) 054 [arXiv: 1309.5093] [INSPIRE].

[23] G. Siopsis and J. Therrien, Analytic Calculation of Properties of Holographic Superconductors, JHEP 05 (2010) 013 [arXiv: 1003.4275] [INSPIRE]. 
[24] A. Donos and J.P. Gauntlett, Holographic helical superconductors, JHEP 12 (2011) 091 [arXiv:1109.3866] [INSPIRE].

[25] A. Donos and J.P. Gauntlett, Helical superconducting black holes, Phys. Rev. Lett. 108 (2012) 211601 [arXiv:1203.0533] [INSPIRE].

[26] R.-G. Cai, L. Li, L.-F. Li and R.-Q. Yang, Introduction to Holographic Superconductor Models, Sci. China Phys. Mech. Astron. 58 (2015) 060401 [arXiv: 1502.00437] [INSPIRE].

[27] Q. Pan, J. Jing and B. Wang, Analytical investigation of the phase transition between holographic insulator and superconductor in Gauss-Bonnet gravity, JHEP 11 (2011) 088 [arXiv: 1105.6153] [INSPIRE].

[28] H.-F. Li, R.-G. Cai and H.-Q. Zhang, Analytical Studies on Holographic Superconductors in Gauss-Bonnet Gravity, JHEP 04 (2011) 028 [arXiv:1103.2833] [INSPIRE].

[29] R. Gregory, S. Kanno and J. Soda, Holographic Superconductors with Higher Curvature Corrections, JHEP 10 (2009) 010 [arXiv:0907.3203] [INSPIRE].

[30] R.-G. Cai, Z.-Y. Nie and H.-Q. Zhang, Holographic p-wave superconductors from Gauss-Bonnet gravity, Phys. Rev. D 82 (2010) 066007 [arXiv:1007.3321] [INSPIRE].

[31] Q. Pan, B. Wang, E. Papantonopoulos, J. Oliveira and A.B. Pavan, Holographic Superconductors with various condensates in Einstein-Gauss-Bonnet gravity, Phys. Rev. D 81 (2010) 106007 [arXiv:0912.2475] [INSPIRE].

[32] R.-G. Cai, Z.-Y. Nie and H.-Q. Zhang, Holographic Phase Transitions of P-wave Superconductors in Gauss-Bonnet Gravity with Back-reaction, Phys. Rev. D 83 (2011) 066013 [arXiv: 1012.5559] [INSPIRE].

[33] L. Zhang, Q. Pan and J. Jing, Holographic p-wave superconductor models with Weyl corrections, Phys. Lett. B 743 (2015) 104 [arXiv:1502.05635] [INSPIRE].

[34] P. Chaturvedi and G. Sengupta, p-wave Holographic Superconductors from Born-Infeld Black Holes, JHEP 04 (2015) 001 [arXiv: 1501.06998] [INSPIRE].

[35] Z. Zhao, Q. Pan and J. Jing, Holographic insulator/superconductor phase transition with Weyl corrections, Phys. Lett. B 719 (2013) 440 [arXiv:1212.3062] [INSPIRE].

[36] J. Jing, Q. Pan and S. Chen, Holographic Superconductor/Insulator Transition with logarithmic electromagnetic field in Gauss-Bonnet gravity, Phys. Lett. B 716 (2012) 385 [arXiv: 1209.0893] [INSPIRE].

[37] G.T. Horowitz and R.C. Myers, The AdS/CFT correspondence and a new positive energy conjecture for general relativity, Phys. Rev. D 59 (1998) 026005 [hep-th/9808079] [INSPIRE].

[38] T. Nishioka, S. Ryu and T. Takayanagi, Holographic Superconductor/Insulator Transition at Zero Temperature, JHEP 03 (2010) 131 [arXiv:0911.0962] [INSPIRE].

[39] E. Witten, Anti-de Sitter space, thermal phase transition and confinement in gauge theories, Adv. Theor. Math. Phys. 2 (1998) 505 [hep-th/9803131] [INSPIRE].

[40] T. Albash and C.V. Johnson, A Holographic Superconductor in an External Magnetic Field, JHEP 09 (2008) 121 [arXiv:0804.3466] [INSPIRE].

[41] X.-H. Ge, B. Wang, S.-F. Wu and G.-H. Yang, Analytical study on holographic superconductors in external magnetic field, JHEP 08 (2010) 108 [arXiv:1002.4901] [INSPIRE]. 
[42] X.-H. Ge and H.-Q. Leng, Analytical calculation on critical magnetic field in holographic superconductors with backreaction, Prog. Theor. Phys. 128 (2012) 1211 [arXiv:1105.4333] [INSPIRE].

[43] S.-1. Cui and Z. Xue, Critical magnetic field in a holographic superconductor in Gauss-Bonnet gravity with Born-Infeld electrodynamics, Phys. Rev. D 88 (2013) 107501 [arXiv: 1306.2013] [INSPIRE].

[44] D. Roychowdhury, Effect of external magnetic field on holographic superconductors in presence of nonlinear corrections, Phys. Rev. D 86 (2012) 106009 [arXiv:1211.0904] [INSPIRE].

[45] T. Albash and C.V. Johnson, Vortex and Droplet Engineering in Holographic Superconductors, Phys. Rev. D 80 (2009) 126009 [arXiv:0906.1795] [InSPIRE].

[46] D. Roychowdhury, Holographic droplets in p-wave insulator/superconductor transition, JHEP 05 (2013) 162 [arXiv: 1304.6171] [INSPIRE].

[47] R.-G. Cai, S. He, L. Li and L.-F. Li, A Holographic Study on Vector Condensate Induced by a Magnetic Field, JHEP 12 (2013) 036 [arXiv: 1309.2098] [INSPIRE].

[48] K. Maeda and T. Okamura, Characteristic length of an AdS/CFT superconductor, Phys. Rev. D 78 (2008) 106006 [arXiv:0809.3079] [INSPIRE].

[49] H.-B. Zeng, Z.-Y. Fan and H.-S. Zong, Superconducting Coherence Length and Magnetic Penetration Depth of a p-wave Holographic Superconductor, Phys. Rev. D 81 (2010) 106001 [arXiv:0912.4928] [INSPIRE].

[50] H.-B. Zeng, Z.-Y. Fan and H.-S. Zong, Characteristic length of a Holographic Superconductor with d-wave gap, Phys. Rev. D 82 (2010) 126014 [arXiv:1006.5483] [InSPIRE].

[51] D. Roychowdhury, Chern-Simons vortices and holography, JHEP 10 (2014) 18 [arXiv:1407.3464] [INSPIRE].

[52] D. Roychowdhury, Towards holographic duals for anomalous supercurrents, arXiv: 1403.0085 [INSPIRE].

[53] K. Maeda, M. Natsuume and T. Okamura, Vortex lattice for a holographic superconductor, Phys. Rev. D 81 (2010) 026002 [arXiv:0910.4475] [INSPIRE].

[54] K. Maeda and T. Okamura, Vortex flow for a holographic superconductor, Phys. Rev. D 83 (2011) 066004 [arXiv: 1012.0202] [InSPIRE].

[55] T. Shiromizu, S. Ohashi and R. Suzuki, A no-go on strictly stationary spacetimes in four/higher dimensions, Phys. Rev. D 86 (2012) 064041 [arXiv:1207.7250] [INSPIRE].

[56] B. Bakon and M. Rogatko, Complex scalar field in strictly stationary Einstein-Maxwell-axion-dilaton spacetime with negative cosmological constant, Phys. Rev. D 87 (2013) 084065 [arXiv:1305.1401] [INSPIRE].

[57] M. Regis, J.-Q. Xia, A. Cuoco, E. Branchini, N. Fornengo and M. Viel, Particle dark matter searches outside the Local Group, Phys. Rev. Lett. 114 (2015) 241301 [arXiv:1503.05922] [INSPIRE].

[58] Y. Ali-Haimoud, J. Chluba and M. Kamionkowski, Constraints on Dark Matter Interactions with Standard Model Particles from Cosmic Microwave Background Spectral Distortions, Phys. Rev. Lett. 115 (2015) 071304 [arXiv:1506.04745] [INSPIRE]. 
[59] J. Bramante and T. Linden, Detecting Dark Matter with Imploding Pulsars in the Galactic Center, Phys. Rev. Lett. 113 (2014) 191301 [arXiv:1405.1031] [INSPIRE].

[60] J. Fuller and C. Ott, Dark Matter-induced Collapse of Neutron Stars: A Possible Link Between Fast Radio Bursts and the Missing Pulsar Problem, Mon. Not. Roy. Astron. Soc. 450 (2015) L71 [arXiv:1412.6119] [INSPIRE].

[61] I. Lopes and J. Silk, A particle dark matter footprint on the first generation of stars, Astrophys. J. 786 (2014) 25 [arXiv:1404.3909] [INSPIRE].

[62] A. Nakonieczna, M. Rogatko and R. Moderski, Dynamical Collapse of Charged Scalar Field in Phantom Gravity, Phys. Rev. D 86 (2012) 044043 [arXiv:1209.1203] [INSPIRE].

[63] A. Nakonieczna, M. Rogatko and E. Nakonieczny, Dark sector impact on gravitational collapse of an electrically charged scalar field, JHEP 11 (2015) 012 [arXiv:1508.02657] [INSPIRE].

[64] A. Geringer-Sameth et al., Indication of Gamma-ray Emission from the Newly Discovered Dwarf Galaxy Reticulum II, Phys. Rev. Lett. 115 (2015) 081101 [arXiv:1503.02320] [INSPIRE].

[65] K.K. Boddy and J. Kumar, Indirect Detection of Dark Matter Using MeV-Range Gamma-Ray Telescopes, Phys. Rev. D 92 (2015) 023533 [arXiv:1504.04024] [INSPIRE].

[66] K. Van Tilburg, N. Leefer, L. Bougas and D. Budker, Search for ultralight scalar dark matter with atomic spectroscopy, Phys. Rev. Lett. 115 (2015) 011802 [arXiv:1503.06886] [InSPIRE].

[67] P. Jean et al., Early SPI/INTEGRAL measurements of $511 \mathrm{keV}$ line emission from the 4th quadrant of the Galaxy, Astron. Astrophys. 407 (2003) L55 [astro-ph/0309484] [INSPIRE].

[68] J. Chang et al., An excess of cosmic ray electrons at energies of 300-800 GeV, Nature 456 (2008) 362 [INSPIRE].

[69] PAMELA collaboration, O. Adriani et al., An anomalous positron abundance in cosmic rays with energies 1.5-100 GeV, Nature 458 (2009) 607 [arXiv:0810.4995] [INSPIRE].

[70] Muon G-2 collaboration, G.W. Bennett et al., Final Report of the Muon E821 Anomalous Magnetic Moment Measurement at BNL, Phys. Rev. D 73 (2006) 072003 [hep-ex/0602035] [INSPIRE].

[71] D. Harvey, R. Massey, T. Kitching, A. Taylor and E. Tittley, The non-gravitational interactions of dark matter in colliding galaxy clusters, Science 347 (2015) 1462 [arXiv: 1503.07675] [INSPIRE].

[72] R. Massey et al., The behaviour of dark matter associated with four bright cluster galaxies in the $10 \mathrm{kpc}$ core of Abell 382\%, Mon. Not. Roy. Astron. Soc. 449 (2015) 3393 [arXiv: 1504.03388] [INSPIRE].

[73] BABAR collaboration, J.P. Lees et al., Search for a Dark Photon in $e^{+} e^{-}$Collisions at BaBar, Phys. Rev. Lett. 113 (2014) 201801 [arXiv:1406.2980] [InSPIRE].

[74] E. Nakonieczny and M. Rogatko, Analytic study on backreacting holographic superconductors with dark matter sector, Phys. Rev. D 90 (2014) 106004 [arXiv:1411.0798] [INSPIRE].

[75] E. Nakonieczny, M. Rogatko and K.I. Wysokinski, Magnetic field in holographic superconductor with dark matter sector, Phys. Rev. D 91 (2015) 046007 [arXiv:1502.02550] [INSPIRE]. 
[76] Ł. Nakonieczny, M. Rogatko and K.I. Wysokiński, Analytic investigation of holographic phase transitions influenced by dark matter sector, Phys. Rev. D 92 (2015) 066008 [arXiv: 1509.01769] [INSPIRE].

[77] M. Rogatko and K.I. Wysokinski, P-wave holographic superconductor/insulator phase transitions affected by dark matter sector, arXiv:1508.02869 [INSPIRE].

[78] Y. Peng, Holographic entanglement entropy in superconductor phase transition with dark matter sector, Phys. Lett. B 750 (2015) 420 [arXiv: 1507.07399] [INSPIRE].

[79] T. Vachaspati and A. Achucarro, Semilocal cosmic strings, Phys. Rev. D 44 (1991) 3067 [INSPIRE].

[80] A. Achucarro and T. Vachaspati, Semilocal and electroweak strings, Phys. Rept. 327 (2000) 347 [hep-ph/9904229] [INSPIRE].

[81] B. Hartmann and F. Arbabzadah, Cosmic strings interacting with dark strings, JHEP 07 (2009) 068 [arXiv:0904.4591] [INSPIRE].

[82] Y. Brihaye and B. Hartmann, The effect of dark strings on semilocal strings, Phys. Rev. D 80 (2009) 123502 [arXiv:0907.3233] [InSPIRE].

[83] Y. Brihaye and B. Hartmann, Holographic superfluid/fluid/insulator phase transitions in 2+1 dimensions, Phys. Rev. D 83 (2011) 126008 [arXiv:1101.5708] [INSPIRE].

[84] H. Davoudiasl, H.-S. Lee and W.J. Marciano, 'Dark' Z implications for Parity Violation, Rare Meson Decays and Higgs Physics, Phys. Rev. D 85 (2012) 115019 [arXiv:1203.2947] [INSPIRE].

[85] H. Davoudiasl, H.-S. Lee, I. Lewis and W.J. Marciano, Higgs Decays as a Window into the Dark Sector, Phys. Rev. D 88 (2013) 015022 [arXiv:1304.4935] [INSPIRE].

[86] K. Freese, M. Lisanti and C. Savage, Colloquium: Annual modulation of dark matter, Rev. Mod. Phys. 85 (2013) 1561 [arXiv: 1209.3339] [INSPIRE].

[87] O. Domenech, M. Montull, A. Pomarol, A. Salvio and P.J. Silva, Emergent Gauge Fields in Holographic Superconductors, JHEP 08 (2010) 033 [arXiv:1005.1776] [INSPIRE].

[88] M. Montull, O. Pujolàs, A. Salvio and P.J. Silva, Magnetic Response in the Holographic Insulator/Superconductor Transition, JHEP 04 (2012) 135 [arXiv:1202.0006] [INSPIRE].

[89] Ó.J.C. Dias, G.T. Horowitz, N. Iqbal and J.E. Santos, Vortices in holographic superfluids and superconductors as conformal defects, JHEP 04 (2014) 096 [arXiv:1311.3673] [INSPIRE]. 